International Journal of Power Electronics and Drive Systems (IJPEDS)

Vol. 12, No. 3, September 2021, pp. 1576 1585

ISSN: 2088-8694, DOI: 10.11591/ijpeds.v12.i3.pp1576-1585

1576

\title{
Newly fault-tolerant indirect vector control for traction inverter
}

\author{
Sara Zerdani, Mohamed Larbi Elhafyani, Hicham Fadil, Smail Zouggar \\ Laboratory of Electrical Engineering and Maintenance (LGEM), Higher School of Technology, University Med First \\ Oujda, Morocco
}

\begin{abstract}
Article Info
Article history:

Received Fev 19, 2021

Revised Mar 1, 2021

Accepted Mar 05, 2021

\section{Keywords:}

Fault diagnosis

Fault-tolerant-control

Four switches inverter

IGBT's fault

PMSM

ABSTRACT

The traction inverter is a crucial power device in the electric vehicle's powertrain, and its failure is intolerable as it would considerably compromise the system's safety. For more reliable driving, installing a traction inverter that is sufficiently resistant to electrical failure is inherent. Due to its compact size and the small number of switches incorporated in three-phase four-switch inverter, this modular topology was used to compensate for the open switch's failure. However, it is known to have manifold weaknesses mainly distinguished in the low-frequency region. This paper introduces a new fault-tolerant indirect control that handles the IGBT's failure constituting the traction inverter. The fault compensator is designed first based on the Proportional Integral regulator combined with the notch filter to mitigate the current imbalance and restore the DC voltage equilibrium.Furthermore, to conceive a comprehensive fault-tolerant control, there must therefore contain an accurate fault detector. In this regard, an uncomplicated fault diagnosis method based on the current spectral analysis has been performed. The effectiveness of the submitted controller was validated by simulation using Matlab.
\end{abstract}

This is an open access article under the CC BY-SA license.

\section{Corresponding Author:}

Sara Zerdani

Laboratory of Electrical Engineering and Maintenance

Higher School of Technology

University Med First Oujda, Morocco

Email: zerdanisara9@gmail.com

\section{INTRODUCTION}

With the tangible increase in oil price and climate change, electric vehicles have emerged as the most promising technological solution by far. In this respect, research and development have made great strides in improving electric powertrain's performance, especially under faulty operating conditions. A great deal of research has suggested incorporating fault tolerant control (FTC) into these systems, making the driving of these vehicles more efficient and safer in the event of damage to any of its components [1].

Seeing that power electronics is the most affected device by the powertrain's electrical faults, it has exclusively occupied several researchers' attention in diagnosis [2] and fault compensation [3]. The open-circuit switch/leg is indicated in reference [4] as the predominant inverter fault type. This malfunction induces uncontrolled direct and quadrature current and hence a high pulsating torque. As a result, it produces a noticeable change in the three current phases. Exploiting this modification, the authors have developed in reference [5] a fault detector based practically on fast fourier transform (FFT) and Support Vector Machine. The Fast Fourier transform was applied to obtain the meaningful current fault signature; subsequently, they adopted the support 
vector machine algorithm to locate the open/short circuit switch. In reference [6], Xing Hu Jian Zhong has suggested an innovative approach to constructing the fault detector. They used the Extended State Observer as the second redundant process calculator. The difference between the actual and the analytical process outputs constitutes the residual that contains fault information concern.

A traction inverter's fault diagnosis can only be completed by reconfiguring the initial topology; a contemporary structure adaptable to the fault conditions is strictly an exigency to maintain system operational continuity [7][8]. In the literature, we distinguish two main categories of fault-tolerant topology (FTT), which are generally classified by the costs and the number of components they are composed. Other criteria are to be taken into accounts to select the adequate FTT that is typically the fault power rating factor (FPRF) and the Current rating of the auxiliary switches. With the redundant phase topology, the overall cost associated with fault-tolerant components is estimated at $233 \%$ of the standard inverter, justified as costly. However, it does guarantee a nominal operating mode identical to the conventional three-phase inverter with an FPRF equal to one. In contrast, the four-switch three-phase (FSTP) inverter has originated to minimize the number of interference circuits and the extra switches integrated into the fault-tolerant topology with the additive leg. In this hardware reconfiguration, the defected motor phase is reconnected to the DC bus's midpoint via the Triac, as indicated in Figure 1.

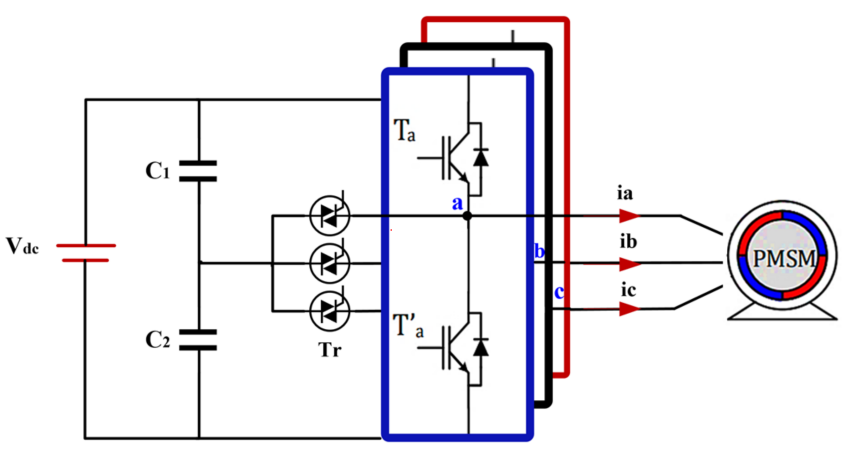

Figure 1. Fault-tolerant traction inverter topology

Despite its benefits, this topology has a cost in terms of worsening system performance. As specified in part number [9], this circuit design's main shortcoming resides in the motor currents phases unbalance resulting from the wide voltage variation across the DC link's two capacitors. A predictive Space Vector Modulation is introduced in reference [10] to overcome this topology's predominant weaknesses and compensate for the DC link voltage ripple. In another way, the DC-link voltage and the currents unbalance are repaired based on double synchronous reference frame PI controllers [11]. As reported in [12], the motor current bothers were solved using a new reference voltage.

This article has ensured the faulty $6 \mathrm{~S} 3 \mathrm{P}$ inverter's operational continuity by modeling the output voltages of the 4S3P inverter and offsetting the voltage fluctuation of the intermediate capacitor circuit. Thus, we outline the fault diagnosis module, which supplements the proposed remedial fault control. It is structured systematically thus: section 2 exposes the mathematical model of the PMSM in (DQ) rotating reference frames under healthy operating conditions and details the Indirect Vector Control that drives the PMSM. A short description of the proposed fault-tolerant control structure is then given in section 3. Next, it highlights the rescue topology's weak points and explains the technical solution proposed to overwork them. After that, it presents a survey of the fault diagnosis technique used throughout this work. Before finally, we will discuss the simulation result of an open-circuit fault compensation approach.

\section{THE PMSM MODEL AND INDIRECT VECTOR CONTROL}

The permanent magnet synchronous motor (PMSM) model is introduced in this section. We have built this model with the following assumptions: the iron losses and saturation of the magnetic circuit are negligible, the Foucault currents and hysteresis phenomena are ignored, and the magnetomotive forces produced in the stator winding are sinusoidally distributed. Also, this part will expose the indirect vector control (IVC) employed to control the Traction Inverter that drives the PMSM. 


\subsection{The model of the PMSM in the (DQ0) rotating reference frame}

The simplified model of the PMSM could obtain by transforming the (ABC) reference frame model described by Pillay, et al. [13] linked to the stator into a model in the (DQ0) rotating frame. The electrical equations are exposed while considering the partial flux derivatives as it follows:

$$
\left[\begin{array}{l}
v_{d}(t) \\
v_{q}(t)
\end{array}\right]=R_{s}\left[\begin{array}{c}
i_{d}(t) \\
i_{q}(t)
\end{array}\right]+\frac{d}{d t}\left[\begin{array}{c}
\Phi_{d}(t) \\
\Phi_{q}(t)
\end{array}\right]+\omega_{r} A\left[\begin{array}{c}
\Phi_{d}(t) \\
\Phi_{q}(t)
\end{array}\right]
$$

Where: $A=\left[\begin{array}{cc}0 & -1 \\ 1 & 0\end{array}\right]$

Only the direct and quadrature current are retained as typically; the homopolar component converges towards zeros while the machine is in normal condition. This factor leads us to overlook the relationship between the flux and the homopolar current, which means that only the direct component, quadrature, and rotor angle are involved. The flux can therefore be expressed in the (DQ0) reference frame by these expressions:

$$
\left\{\begin{array}{l}
\Phi_{d}(t)=\Psi_{m}-L_{d} i_{d}(t) \\
\Phi_{q}(t)=L_{q} i_{q}(t)
\end{array}\right.
$$

Where: $\mathrm{L}_{d}=\mathrm{L}_{s 0}-\mathrm{L}_{m 0}+\frac{\mathrm{L}_{s 2}}{2}+\mathrm{L}_{m 2}$ and $\mathrm{L}_{q}=\mathrm{L}_{s 0}-\mathrm{L}_{m 0}-\frac{\mathrm{L}_{s 2}}{2}-\mathrm{L}_{m 2}$

$\mathrm{L}_{s 0}$ and $\mathrm{L}_{m 0}$ are the average values of the proper and mutual inductances, $\mathrm{L}_{s 2}$ and $\mathrm{L}_{m 2}$ are the amplitudes of the sinusoidal components. From as shown in (1) and (2) the PMSM simplified model is described as follow:

$$
\left\{\begin{array}{l}
\mathrm{v}_{d}(t)=R_{s} i_{d}(t)+L_{d} \frac{d i_{d}(t)}{d t}-L_{q} \omega_{r} i_{q}(t) \\
\mathrm{v}_{q}(t)=R_{s} i_{q}(t)+L_{q} \frac{d i_{q}(t)}{d t}+L_{d} \omega_{r} i_{d}(t)+\omega_{r} \Psi_{m}
\end{array}\right.
$$

It is clear from as shown in (3) that the model of the machine in the (DQ0) rotating reference frame resulted in fewer equations, variables, and derivatives compared to the model developed in the (ABC) reference frame (see reference [13]). Nevertheless, it is observable that this reference frame presents a non-linearity because of the linkage between the direct and quadrature axes.

Thus, the electromagnetic torque is determined in terms of direct current and quadrature of the PMSM in the (DQ0) rotating reference frame by the following equation:

$$
\mathrm{T}_{e m}=\frac{3}{2} P i_{q}(t)\left\{\Psi_{m}+\left(L_{d}-L_{q}\right) i_{d}(t)\right\}
$$

Due to the conceptual clarity obtained with a single set of two windings on the stator, this transformation has an obvious benefit for performing vector control. This kind of control requires the state space equation of the PMSM. From the electrical model outlined as shown in (3) and the mechanical model (4), the PMSM inputs models are respectively the voltages $\mathrm{v}_{d}, \mathrm{v}_{q}$ and the load torque $\mathrm{T}_{\text {load }}$, while the outputs are the speed and electromagnetic torque $\mathrm{T}_{e m}$. The non-linear state model of the PMSM is exposed in the (DQ0) rotating reference frame by the following state-space model [14]:

$$
\left\{\begin{array}{l}
x=A \dot{x}+B u \\
y=C x
\end{array}\right.
$$

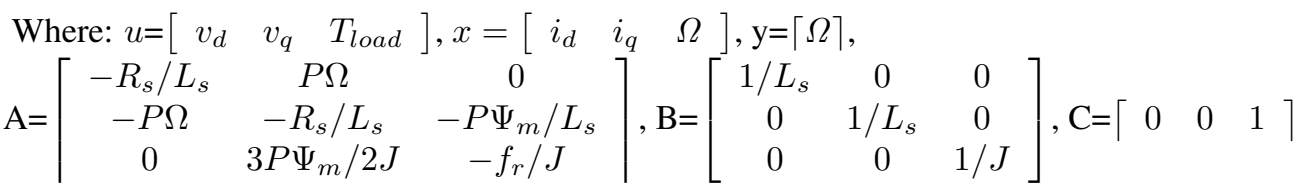

Hence, to get the maximum torque, the direct current is forced to be zero in the Vector Control so that the expression of the developed electromagnetic torque in the state space model could be reduced. 


\subsection{PMSM indirect vector control}

In this part, the PM Synchronous Motor's vector control is directly derived from its dynamic model developed in section 2.1. The torque's decoupling and the mutual flux one against the other are referred to as vector control (VC). This approach incorporates the current controllers of the PMSM and, which are mostly the Proportional and Integral controller. As illustrated in the asymptotic schematic of the VC showed in Figure 2, the estimated direct current $i_{d}$ and quadrature current $i_{q}$ are compared to their reference, which is respectively the zero value and the $i_{q}{ }^{r e f}$, resulting from the speed controller output. Then the proper errors of the currents are transmitted to the PI controllers, which generate the inputs of the decoupling block and which in its role produces the controlled stator voltage components $v_{d}^{r e f}$, and $v_{q}^{r e f}$. The equations express the reference voltage vectors are:

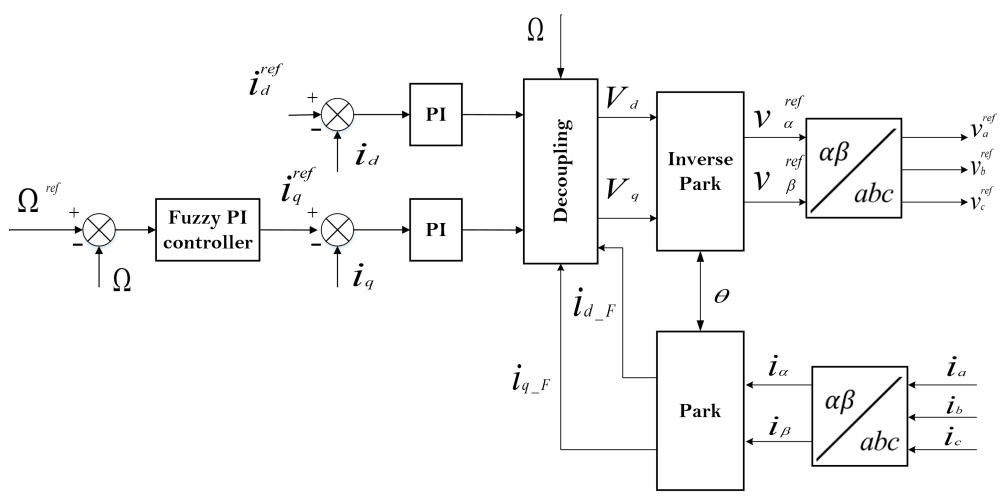

Figure 2. Schematic diagram of an indirect vector control for PMSM

$$
\left\{\begin{array}{l}
v_{d}^{r e f}=\left(K_{p_{-i d}}+K_{i_{i} i d} \frac{T_{s} z}{z-1}\right)\left(i_{d}{ }^{*}-i_{d}\right)-i_{q} L_{s} P \Omega \\
v_{q}^{r e f}=\left(K_{p_{-i q}}+K_{i_{\imath i q}} \frac{T_{s} z}{z-1}\right)\left(i_{q}{ }^{*}-i_{q}\right)+i_{d} L_{s} P \Omega+\psi_{m} P \Omega
\end{array}\right.
$$

Where: $K_{p_{-i d}}, K_{i_{-} i d}, K_{p_{-i q}}$ and $K_{i_{-} i q}$ are respectively the direct and the quadrature current PI gains. A further target objective of the Indirect Vector Control (IVC) is to track the reference speed; for this reason, the Fuzzy PI controller is implemented in the IVC schematic. It is to be noted that such controls remain valid only when the machine is in a sound operating condition. In the case of IGBT's open circuit fault, it has been found necessary to modify this configuration while adding a new fault control law [15].

\section{FAULT-TOLERANT IVC OVERALL}

This section briefly describes the proposed Fault-Tolerant Control's working principle and then details each unit's functionality. In various proposals, the SSTP controller was designed mostly based on two major trends: direct torque control and indirect vector control. In neither configuration, the system remains unstable without a fault compensation unit and new materiel reconfiguration at IGBT's open-circuit fault, thus requiring an updated control algorithm capable of maintaining acceptable system performance [16][17]. The back-up topology employed in this work to remediate one or two open IGBT power devices is depicted in Figure 1.

Furthermore, the control strategy adopts as a principle the inclusion of a new voltage compensation component to the reference voltage generated by the classical Vector Control. A notch filter was implemented to remove the second-order harmonic from the stator currents and the DC capacitor's voltage sources. As depicted in Figure 3, the first notch filter's inputs are the quadrature and the direct current. Moreover, the second filter receives the DC bus capacitor's voltage as input. The offset suppression's bloc responds actively to the occurrence of the fault. According to Figure 3, the fault diagnosis module provides the foremost system state information to all FTC subsystems. This unit generates residuals that localize the open IGBT's power device position through the stator currents' spectral analysis.

Once the fault detector evaluates the residues, it calculates the logical decision and subsequently isolates the faulty leg while suppressing the opposite IGBT power device's signal command that corresponds to the same leg. Then, the Triac is controlled to link the midpoint of the DC link capacitors to the isolated motor 
phase; in our case, this was the $\mathrm{C}$ motor phase. After the step mentioned above, the diagnostic unit activates the offset suppression's bloc. The proposed strategy's vital interest is its ability to maintain acceptable system characteristics, even after the fault occurs, without degrading the system's overall performance under healthy operating conditions [18][19]. This point has not been taken into account by the passive FTC theory.

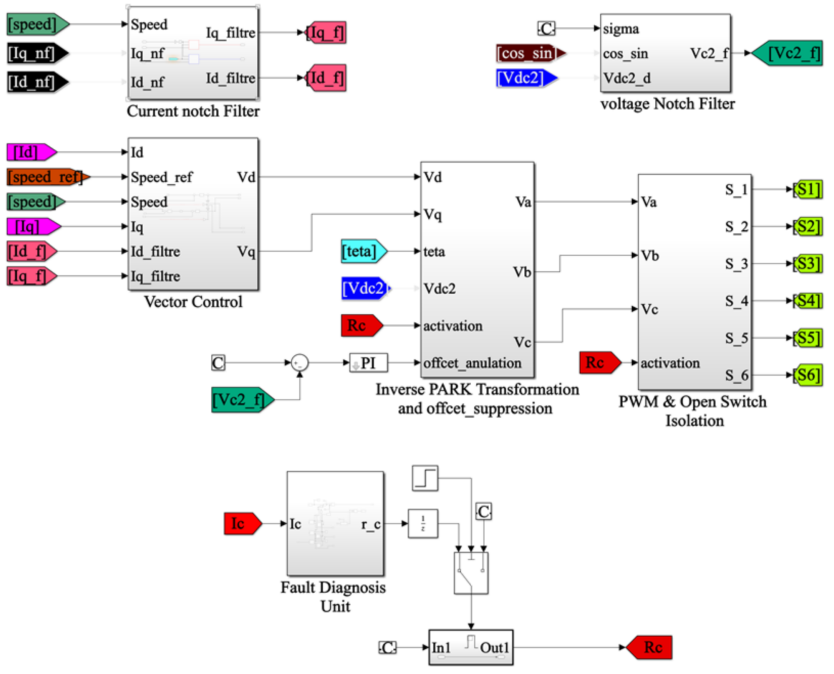

Figure 3. Fault-Tolerant IVC scheme for PM synchronous motor drive

\subsection{Restructuration strategy}

This section examines the impact of the open IGBT's failure on PMSM's pre-set model closely. We will explain how the three-phase four-switch inverter can be a solution to reconfigure the Six-Switch topology at the occurrence of the studied fault. Indeed, this type of converter has recently attracted several authors' attention because of the reduced number of switches that fit in its construction and thus allows to power threephase motors such as the PMSM, see references (Errabelli, et al. [20] and Freire, et al. [21]). These works have confirmed experimentally that such an inverter has limited performance, especially in the low-frequency range. According to references [22][23], the main reason for the fluctuation of the intermediate circuit voltage is the behavior of the capacitive impedance of the $\mathrm{C}$ phase, which is mainly used to replace the defective branch. This behavior can be explained by adding the voltage's fluctuating component to the voltage's direct part.

$$
\left\{\begin{array}{l}
u_{d c 1}=V_{d c} / 2-V^{\prime}{ }^{\prime} \\
u_{d c 2}=V_{d c} / 2+V^{\prime}{ }_{c}
\end{array}\right.
$$

Where: $V_{c}^{\prime}=-1 / 2 C \int i_{c 1} d t$

Another factor that affects the TPFS inverter's effectiveness is the voltage offset at the DC link's midpoint. In reference [24], the authors state in their paper that the offset component in the mean voltage of the DC link is the principal reason causing the DC components to appear in the PMSM load current. In the (ABC) reference frame, we could delineate this result by including the DC component in the stator current equation.

$$
\left\{\begin{array}{c}
i_{s a}=i_{s a}^{u f}+i_{s a}^{h}-i_{0} \\
i_{s b}=i_{s b}^{u f}+i_{s b}^{h}-i_{0} \\
i_{s c}=i_{s c}^{u f}+i_{s c}^{h}-2 i_{0}
\end{array}\right.
$$

Where: $i_{s x}^{u f}, i_{s x}^{h}$ and $i_{0}$ are respectively the unfaulty current, AC harmonics components, and the DC component current. Hence, it was ascertained by Wang, et al.[18] that the midpoint voltage DC-link $\Delta u$ is linked with the stator current corresponding to the defected leg according as shown in:

$$
\Delta u(t)=\Delta u(t)^{u f}+\Delta u(t)^{h}-i_{0} / C t+\Delta u(0)
$$

With: $\Delta u(t)^{u f}$ and $\Delta u(t)^{h}$ are sequentially the fundamental and the harmonic of the midpoint voltage DC- 
link. To begin examining the additional inspection work required to compensate for the defect result, we have first designated the AC component by $\widetilde{\Delta u}$ and $\overline{\Delta u}$ the compensation element. The $\widetilde{\Delta u}$ included harmonic components in which the second one had to be filtered out. The $\overline{\Delta u}$ part contains the portion $\mathrm{i}_{0} / \mathrm{C}$ amount, which varies as a function of time. To remove the offset voltage component, the PI controller is introduced after the vector control which regulates the portion generated by $i_{0}$ (see Figure 4). We have modeled the PMSM/ (4S3P) inverter system at a standstill by these equations:

$$
\left\{\begin{array}{c}
u_{a c}(t)=R_{s} i_{a}(t)+L_{s} \frac{d i_{a}(t)}{d t}-R_{s} i_{c}(t)-L_{s} \frac{d i_{a}(t)}{d t} \\
u_{b c}(t)=R_{s} i_{b}(t)+L_{s} \frac{d i_{b}(t)}{d t}-R_{s} i_{c}(t)-L_{s} \frac{d i_{a}(t)}{d t} \\
i_{a}(t)=-2 C \frac{d v_{c}(t)}{d t}
\end{array}\right.
$$

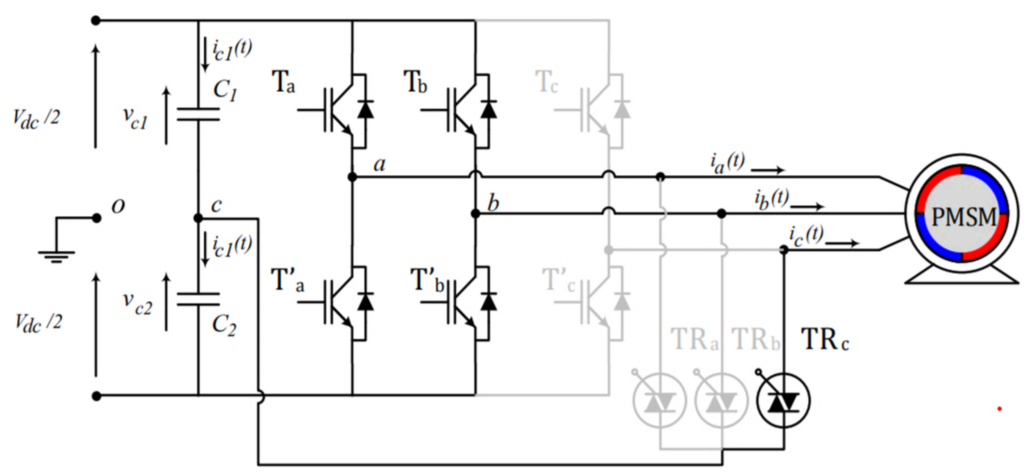

Figure 4. Four-Switch Tree-Phase Inverter Topology

The voltages generated between phases can be expressed in terms of voltage's fluctuating as follows:

$$
\left\{\begin{array}{c}
u_{a c}=u_{a c^{r e f}}{ }^{r e V_{c}^{\prime}} \\
u_{b c}=u_{b c}{ }^{r e f}-V_{c}^{\prime} \\
u_{a b}=u_{a b}{ }^{r e f}-u_{b c}{ }^{r e f}
\end{array}\right.
$$

By rearrangement of (10) and (11), we obtained:

$$
u_{a c}^{r e f}(t)+u_{b c}^{r e f}(t)=2 v_{c}(t)+6 R_{s} C \frac{d v_{c}(t)}{d t}+6 R_{s} L_{s} \frac{d^{2} v_{c}(t)}{d t^{2}}
$$

The PI controller is then sized using the pole compensation method. The open-loop transfer function given as follows:

$$
v_{c}(t)=\frac{k}{2 s}(\tau s+1) \frac{u_{a c}^{r e f}(t)+u_{b c}^{r e f}(t)}{\left(s / s_{1}+1\right)\left(s / s_{2}+1\right)}
$$

Where: $s_{1}$ and $s_{2}$ are the system's poles, and $\mathrm{k}$ is the PI controller's gain.

The operator $\tau$ has chosen to eliminate the dominant pole of the system. The locus-root diagram is drawn to check the system's stability and identify this performance over the whole range of the operator k. For shortness, we are limited to displaying just the PI controller gains in Table 1. Besides, a notch filters are introduced in the control philosophy to eliminate the oscillating component in the stator currents and voltages of the stator DC bus. The filter that concerns the stator current aims to extract the ripple, which results from the second harmonic, accumulating in the form of a signal with the pulsation $2 \omega_{r}$. The following transfer function can identify this notch filter:

$$
\frac{i^{N F}(s)}{i(s)}=\frac{S^{2}+4 \omega_{c}^{2}}{S^{2}+K S+4 \omega_{c}^{2}}
$$

Where $\mathrm{k}$ is gain, which determines the width of the rejection band, $\omega_{c}$ is the notch's center frequency (the frequency of the harmonic to be attenuated). 


\subsection{Open switch/one leg inverter fault diagnosis}

In this work, the fault detector is considered an irreplaceable unit. It is in charge of performing the following subtasks: firstly, it detects the fault and provides the necessary information on the fault location by specifying which switch is affected. Secondly, it separates and isolates the faulty branch by interrupting the control signal from the opposite switch that belongs to the same faulty part. Third, it activates the control law used to compensate for the failure. Since such a failure introduces an observed change in the current phase, some authors have taken advantage of the power spectral density combined with the machine learning algorithm tool to extract and classify the fault characteristics [25]. This type of detector can lead to detection that is more accurate but comes at computational complexity. We used a simple method to identify the defective leg and thus locate the defective switch. This method depends on the variation of the DC and the fundamental magnitude current. In the case of upper or lower open IGBT's fault, the DC current is by definition different from zero and approximately equal to $\pm I_{s} / \pi$ depending on the switch concerned. However, when a wide leg is faulty, this magnitude becomes zero; it is the same as in the healthy operating state. On the other hand, the failure of a complete leg forces the current fundamental magnitude to converge to zero, which is the opposite of the standard case.To quantify these two quantities, the Fourier transform is applied on a window of the current signal during one fundamental frequency cycle. The magnitude of the current fundamental is estimated based on as shown in:

$$
\left|i_{f}\right|=\frac{2}{T} \sqrt{\left(\int_{t-T}^{t} i(t) * \cos (w t) d t\right)^{2}+\left(\int_{t-T}^{t} i(t) * \sin (w t) d t\right)^{2}}
$$

Where: $T=\frac{1}{f_{1}}$ and $f_{1}$ is the current fundamental frequency. On the other hand, the DC currents component is calculated based on as shown in:

$$
i_{d c}=\frac{2}{T} \int_{t-T}^{t} i(t) d t
$$

By comparing the normalized computed components to a fixed threshold, it is possible to define the residual that detects the defected leg as [26]:

$$
\mathrm{r}_{\mathrm{a}, \mathrm{b}, \mathrm{c}}^{\mathrm{f}}=\left\{\begin{array}{cr}
1 & \text { if }\left\|\frac{\mathrm{i}_{\mathrm{n}}^{\mathrm{f}}-\mathrm{i}_{\mathrm{m}}^{\mathrm{f}}}{\max \left(\mathrm{i}_{\mathrm{a}}^{\mathrm{f}}, \mathrm{i}_{\mathrm{b}}^{\mathrm{f}}, \mathrm{if}_{\mathrm{c}}^{\mathrm{f}}\right)}\right\|>\mathrm{T}_{\mathrm{r}, 1} \\
0 & \text { Otherwise }
\end{array}\right.
$$

Where: $\mathrm{n}$ and $\mathrm{m}$ are respectively $\{\mathrm{a}, \mathrm{b}, \mathrm{c}\}$ and $\{\mathrm{b}, \mathrm{c}, \mathrm{a}\}$.

Furthermore, to detect and locate the faulty switch, three cases must be checked at each iteration as follow:

$$
\mathrm{r}_{\mathrm{a}, \mathrm{b}, \mathrm{c}}^{\mathrm{dc}}=\left\{\begin{array}{lrr}
1 & \text { if } & \left\|\mathrm{I}_{\mathrm{n}}^{\mathrm{dc}}\right\|>\mathrm{T}_{\mathrm{r}, 2} \\
-1 & \text { if } & \left\|\mathrm{I}_{\mathrm{n}}^{\mathrm{dc}}\right\|>\mathrm{T}_{\mathrm{r}, 3} \\
0 & & \text { Otherwise }
\end{array}\right.
$$

Where $T_{r, 1}, T_{r, 2}, T_{r, 3}$ are fixed thresholds; chosen so that the algorithm could be faster as possible in detecting the failure. Once computing the first residual, a binary logic decision is developed by the fault detector to establish the final residuals $R_{a}, R_{b}, R_{c}$ (see Figure 3). Each one of these thresholds contains information about the state of the corresponding leg.

\section{SIMULATION RESULTS AND DISCUSS}

This section shows by simulation the validity of the control and reconstruction strategy described in section 2. Several simulations are performed in order to test, on the one hand, the ability of the fault diagnosis unit to detect the failures that occur in the branch corresponding to phase $\mathrm{C}$, including the open upper switch and the failure of the entire branch. On the other hand, these simulations are intended to evaluate the proposed control law's effectiveness in compensating for the fault. The control part is built under the MATLAB environment and the power portion in the PSIM software. Note all simulations is runed with the experimental parameters listed in Table 1. In the following analysis, we discuss the simulation results of three cases; the first case aims at systematically observing the behavior of the Active Fault Tolerant Control according to the following hypotheses:

- A latency of $120 \mathrm{~ms}$ is considered between each reconfiguration process

- The motor operates in a low-frequency region, especially at a fixed mechanical speed of $200 \mathrm{rpm}$. 
Under normal operating conditions, the (VC) reasoning drives the system, and the speed profile is adjusted based on the PI controller. By analyzing the simulation results from a general perspective, this controller provides an acceptable steady-state tracking response. However, when the fault is injected, strong ripples are picked up in the electromagnetic torque and undesirable oscillations in the mechanical speed, as shown in Figures 5 (a) and (b). On the other hand, when the fault diagnosis unit is allowed to connect the motor's faulty phase to the midpoint of the DC capacitor bus at the instant $3.62 \mathrm{~s}$, the pulses in the electromagnetic torque remain, but with reduced severity, the same applies to the speed profile.
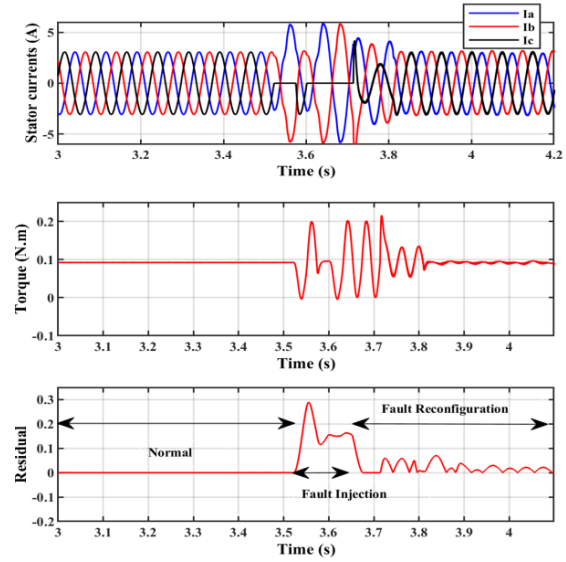

(a)
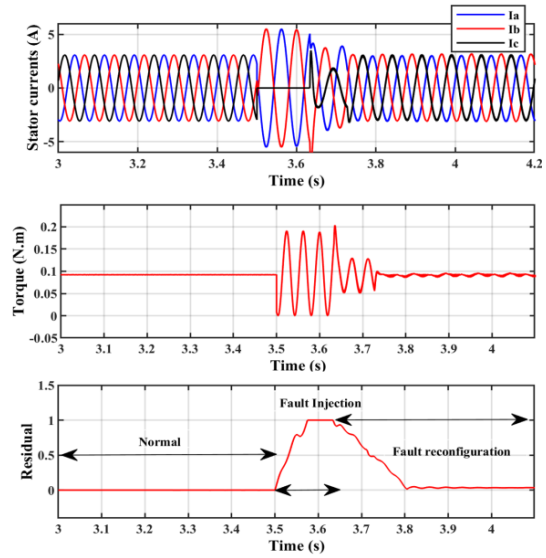

(b)

Figure 5. Response of the system under both; (a) upper open switch fault; (b) open leg fault

After applying the control law described in 3.1, the system recovers and completes a marked reduction in torque and speed oscillation. As a result, the three current phases balance each other.From a particular point of view, the residual in Figure 5 (a) exceeds half of its normalized DC value when the fault is injected. However, when the fault detection unit isolates the fault, it drops to stabilize at zero after the software reconfiguration is triggered. It takes a long time for the residual to reach the fullness of its normalized fundamental value with a deteriorated branch, as shown in Figure 5 (b).

To assess the fault compensation strategy's effectiveness, we have simulated the system spontaneously without external intervention. Despite the transient state error caused by the failure, the fault detection has successfully performed all the previously mentioned subtasks; moreover, the additive control law has reacted at the right time to compensate for a complete branch's failure. Besides, the torque response has become smoother than before.From Figure 6, it can be seen that the proposed fault compensator has the potential to ensure reliable performance even after the occurrence of the fault.
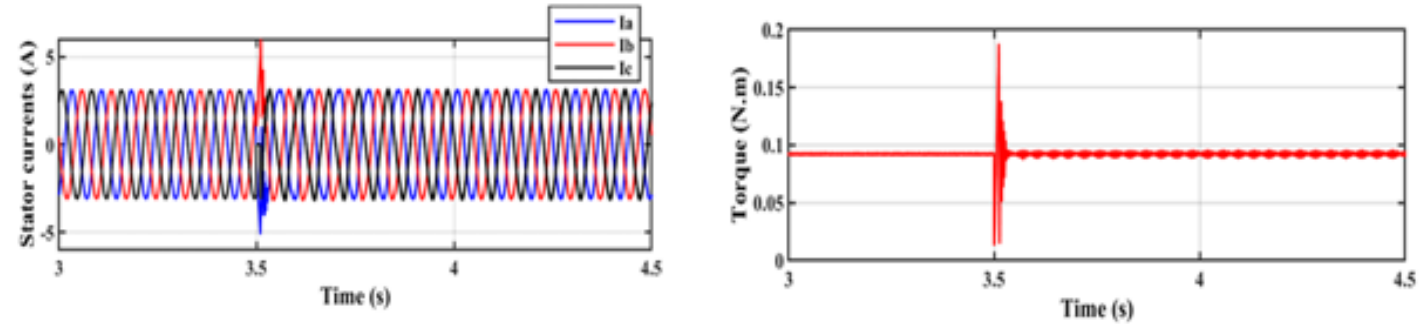

Figure 6. Response of the system with the proposed fault tolerant control strategy

Finally, in the third case, different values are assigned to the reference speed after the fault occurred to evaluate the fuzzy PI controller's flexibility to follow the reference even under undesired conditions. The reference speed curve changes from $200 \mathrm{rpm}$ to $1000 \mathrm{rpm}$ and subsequently stabilizes at $200 \mathrm{rpm}$. It appears from the result entered in Figure 7 (a) that the proposed controller correctly monitored the speed profile under 
both non-faulty and faulty operating conditions. At both the acceleration and deceleration of the reference speed, the controller performed exceptionally well.

By integrating the proposed control law in the IVC, we notice that the quadrature current as well the direct current have both seen a remarkable change in terms of ripples attenuation; this is displayed by Figures 7 (b)and (c). We concluded that the PI controller showed a remarkable result in off setting the DC component as shown in Table 1. It is also to be noted that the notch filter presented an observed image of the currents, although it was imperceptibly affected for low speeds, mainly caused by the ABC current's shape.

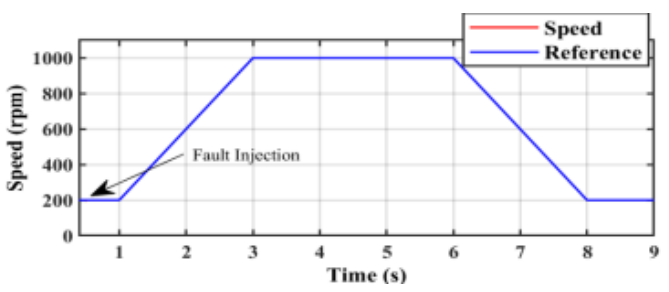

(a)

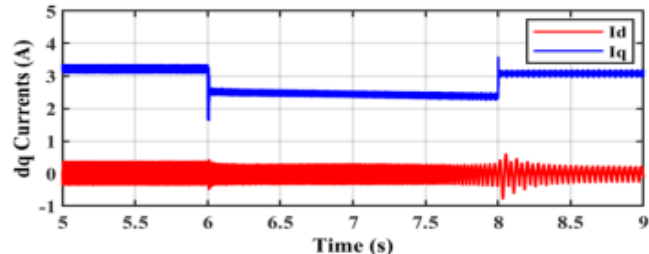

(b)

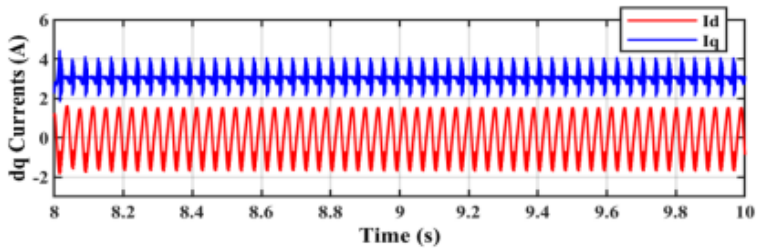

(c)

Figure 7. FTC evaluation results with fuzzy PI speed Controller under different speed reference values

Table 1. Motor parameters

\begin{tabular}{cccc}
\hline Components & Values & Components & Values \\
\hline Rated power & $P=80 \mathrm{~W}$ & Pole pairs & 4 \\
Rated speed & $4000 \mathrm{rpm}$ & Viscous friction & $\mathrm{fr}=0,41010^{-3} \mathrm{Kgm}^{2}$ \\
DC voltage & $24 \mathrm{~V}$ & dc-link capacitor & $4400 \mu \mathrm{F}$ \\
Resistance and inductance & $R=0,43 \Omega, L=1,35 \mathrm{mH}$ & Rotation inertia & $J=0,510^{-3} \mathrm{Nm} / \mathrm{rad}$ \\
\hline
\end{tabular}

\section{CONCLUSION}

This article proposed an adequate strategy for controlling the traction inverter in the event of an open circuit switch fault. The introduced approach is centered on the philosophy of active fault tolerant control. In this approach, the fault diagnosis unit plays a pivotal role in maintaining proper operation during the post fault phase. The latter was developed by analyzing the current spectrum of the defected motor phase. The result has demonstrated the employed fault detector's ability to recognize both the open half leg and the full leg fault. Moreover, it illustrated that the fault detection block is fast sufficient to detect and ensure the hardware and the software reconfiguration.

The four switch-three phase scheme was adopted as fault-tolerant inverter topology. It was driven by the standard vector control associated with an additive control law that responds actively to the fault. This compensator unit is founded principally on the PI controller, combined with notch filters. It performs torque ripple mitigation and stator currents unbalance rectification caused by the DC bus voltage fluctuation. Despite the low voltage compensator's poor achievement in a transient faulty state, it has demonstrated satisfactory results in keeping sound outputs systems.

A constant operation and exceptional speed monitoring for different reference points is achieved at both healthy and faulty operating conditions. The recommended fault-tolerant control strategy exposes a stable system performance. It exhibits more assets such as lower switch numbers and interference circuits and cost reduction reached due to the limited integrated sensors. Such control has to be verified experimentally; in this perspective, we signal that we will validate by test the present work. 


\section{REFERENCES}

[1] K. Lee and M. Lee, "Fault-Tolerant Stability Control for Independent Four-Wheel Drive Electric Vehicle under Actuator Fault Conditions," IEEE Access, vol. 8, pp. 91368-91378, 2020, DOI: 10.1109/ACCESS.2020.2994530.

[2] M. Skowron, T. Orlowska-Kowalska, M. Wolkiewicz, and C. T. Kowalski, "Convolutional neural network-based stator current data-driven incipient stator fault diagnosis of inverter-fed induction motor," Energies, vol. 13, no. 6, 2020, DOI: 10.3390/en13061475.

[3] K. H. Chao, L. Y. Chang, and F. Q. Xu, "Three-Level T-Type Inverter Fault Diagnosis and Tolerant Control Using Single-Phase Line Voltage," IEEE Access, vol. 8, pp. 44075-44086, 2020, DOI: 10.1109/ACCESS.2020.2978141.

[4] Q. Zhu, Z. Li, X. Tan, D. Xie, and W. Dai, "Sensors fault diagnosis and active fault-tolerant control for pmsm drive systems based on a composite sliding mode observer," Energies, vol. 12, no. 9, 2019, DOI: 10.3390/en12091695.

[5] T. Wang, J. Qi, H. Xu, Y. Wang, L. Liu, and D. Gao, "Fault diagnosis method based on FFT-RPCA-SVM for Cascaded-Multilevel Inverter,"ISA Trans, pp. 1-8, 2015, DOI: 10.1016/j.isatra.2015.11.018.

[6] K. Yan, M. Chen, Q. Wu, and B. Jiang, "Extended state observer-based sliding mode fault-tolerant control for unmanned autonomous helicopter with wind gusts," IET Contr. Theory Appli., no. 1, pp. 1-14, 2019.

[7] Wang J, Zhang W, Jiang W, Ma M, Zhang Q, Huang X "Application Ranges of Fault-Tolerant Control for T-Type Three-Level Inverter under Single/Multi-phase Open-Circuit Faults of Inner Switches," IEEE Access, vol. 8, pp. 207599-207609, 2020, DOI: 10.1109/ACCESS.2020.3034919.

[8] M. B. de R. Corrêa, C. B. Jacobina, E. R. C. da Silva, and A. M. N. Lima, "An induction motor drive system with improved fault tolerance,"IEEE Trans. Ind. Applicat., vol. 37, pp. 873-879, 2001, DOI: 10.1109/28.924770.

[9] P. Enjeti and W. Shireen, "A new technique to reject dc-link voltage ripple for inverters operating on programmed PWM waveforms," IEEE Trans. Power Electron., vol. 7, pp. 171-179, 1992, DOI: 10.1109/63.124589.

[10] I. M. Alsofyani, S. M. Kim, and K. B. Lee, "Finite set predictive torque control based on sub-divided voltage vectors of PMSM with deadbeat control and discrete space vector modulation," IEEE Appl. Power Electron., vol. 2019, pp. 1853-1857, 2019, DOI: 10.1109/APEC.2019.8722133.

[11] F. Filipović, M. Petronijević, N. Mitrović, B. Banković, and V. Kostić, "A novel repetitive control enhanced phaselocked loop for synchronization of three-phase grid-connected converters," Energies, vol. 13, no. 1, 2019.

[12] D. C. Meeker and M. J. Newman, "Indirect vector control of a redundant linear induction motor for aircraft launch," Proc. of IEEE, 2009, vol. 97, no. 11, pp. 1768-1776, DOI: 10.1109/JPROC.2009.2030232.

[13] P. Pillay and R. Krishnan, "Modeling of Permanent Magnet Motor Drives," IEEE Trans. Ind. Electron. vol 35, pp.537-541, 1988, DOI: 10.1109/41.9176.

[14] Boumous Z Boumous S, "New Approach in the Fault Tolerant Control of Three-Phase Inverter Fed Induction Motor," Eng. Tech. Appl. Sci. Res., vol. 31, no. 7, pp. 6504-6509, 2020 , DOI: 10.48084/etasr.3898.

[15] R. Wang, J. Zhao, and Y. Liu, "A comprehensive investigation of four-switch three-phase voltage source inverter based on double Fourier integral analysis," IEEE Trans. Power Electron., vol. 26, no. 10, pp. 2774-2787, 2011, DOI: 10.1109/TPEL.2011.2119381.

[16] Li K, Chen C, Cheng S, Yu T, Xiang C, Wu X, See fewer, "A novel fault-tolerant control technique for an inverter with hysteresis current," Computing in Science and Engineering, vol. 21, pp. 92-102, 2019, DOI: 10.1109/MCSE.2018.2886194.

[17] Wu Y. Deng and Z. Wang Y., "Direct slip linear control of three-phase induction motor with one phase open-circuit fault based on three-level inverter," Advanced Materials Research., vol. 433-440, pp. 6776-6782, Jan. 2012.

[18] Gupta Y. and Rao S., "A modified inverter topology for fault-tolerant direct torque control induction motor drive," International Journal of Electronics., vol 107, pp. 1985-2005, 2020, DOI: 10.1080/00207217.2020.1756445.

[19] Boumous Z Boumous S, "New Approach in the Fault Tolerant Control of Three-Phase Inverter Fed Induction Motor," Eng. Tech. Appl. Sci. Res., vol. 31, no. 7, pp. 6504-6509, 2020 , DOI: 10.48084/etasr.3898.

[20] R. R. Errabelli and P. Mutschler, "Fault-tolerant voltage source inverter for permanent magnet drives," IEEE Trans. Power Electron., vol. 27, no. 2, pp. 500-508, 2012.

[21] N.M.A. Freire and A. J. Marques Cardoso, "A fault-tolerant direct controlled PMSG drive for wind energy conversion systems," IEEE Trans. Ind. Electron., vol. 61, no. 2, pp. 821-834, Feb. 2014.

[22] Jaehong Kim, Jinseok Hong, and Kwanghee Nam, "Current Distortion Compensation Scheme for Four-Switch Inverters". IEEE Transactions on Power Electronics. vol 24, pp. 1032-1040, 2009, DOI: 10.1109/TPEL.2008.2011552.

[23] Z. Zeng, W. Zheng, and R. Zhao, "Performance Analysis of the Zero-Voltage Vector Distribution in Three-Phase Four-Switch Converter Using a Space Vector Approach," IEEE Trans. Power Electron., vol. 32, no. 1, pp. 260-273, 2017, DOI: 10.1109/TPEL.2016.2532477

[24] H. Fadil, et al., "Four-Switch Three-Phase PMSM Converter with Output Voltage Balance and DC-Link Voltage Offset Suppression," Information, vol. 8, no. 11, 2017, DOI: 10.1109/CISTEM.2016.8066794.

[25] S. Zerdani, M. L. Elhafyani, and S. Zouggar "Traction Inverter fault detection method based on Welch and K-Nearest Neighbor algorithm," Proc. Springer LNEE, volume 681, pp. 411-419, 2020, DOI:10.1007/978-981-15-6259-44.

[26] H. Fadil, M. L. Elhafyani, and S. Zouggar, "Enhanced Three-Phase Inverter Fault Detection and Diagnosis ApproachDesign and Experimental Evaluation,” vol. 9, no. 2, pp. 559-570, 2018, DOI:10.11591/ijpeds.v9.i2.pp.559-570. 